\title{
A Mathematical Model Analysis for the Transmission Dynamics of HIVIAIDS with Control Strategy
}

\author{
Bogale Assefa Belayneh ${ }^{1}$, Purnachandra Rao Koya ${ }^{2}$ \\ ${ }^{1}$ Department of Mathematics, Debre Berhan University, Debre Berhan, Ethiopia \\ ${ }^{2}$ Department of Mathematics, Wollega University, Nekemte, Ethiopia
}

Email address:

bogaleas@gmail.com (B. A. Belayneh),drkpraophd@gmail.com (P. R. Koya)

\section{To cite this article:}

Bogale Assefa Belayneh, Purnachandra Rao Koya. A Mathematical Model Analysis for the Transmission Dynamics of HIV/AIDS with Control Strategy. Mathematical Modelling and Applications. Vol. 6, No. 3, 2021, pp. 56-69. doi: 10.11648/j.mma.20210603.11

Received: July 30, 2021; Accepted: August 13, 2021; Published: August 23, 2021

\begin{abstract}
This paper examines the transmission dynamics of HIV infection with public health intervention strategies treatment and awareness on the proper procedure of ART treatment. For the problem, a deterministic mathematical model is proposed and analysed qualitatively using the concept of stability of differential equations. The effective reproduction number is computed in terms of model parameters. The existence and stability of disease free and endemic steady states are recognized. The disease free and endemic equilibria are indicated to be locally and globally asymptotically stable whenever the effective reproduction number is less than unity and greater than unity respectively. This means that, HIV infection will die out in the community when the effective reproduction number is less than the threshold value and persist otherwise. Based on the sensitivity analysis of the effective reproduction number, we found that the rate of ART treatment and the rate of awareness on the proper procedure of ART are influential in reducing the magnitude of the reproduction number and thus they are important in decreasing the number of infected population. Numerical simulations support our analytical results that implementing ART treatment at every stage of HIV/AIDS had high impact in reducing the infected population than implementing on a single stage for those who follow the proper procedure of ART treatment. It also verifies the positive impact of awareness on the proper procedure of ART treatment in reducing infected individuals by reducing treatment waning rate. Therefore, our result suggests that ART treatment should be implemented together with awareness on the proper procedure of ART treatment to control the spread of HIV in the community.
\end{abstract}

Keywords: Antiretroviral Treatment, HIV/AIDS Dynamics, Horizontal Transmission, Reproduction Number, Stability

\section{Introduction}

Human immunodeficiency virus (HIV) infection is a disease of immune system caused by HIV virus and it is transmitted primarily via unprotected sexual intercourse, contaminated blood transfusions (Horizontal transmission) and from mother to child during pregnancy, delivery or breastfeeding (Vertical transmission). After entering the body, the virus causes acute infection, which often manifests itself with flue like symptoms and this acute infection is followed by a long asymptomatic (chronic stage) period. With no public health interventions, as the illness progresses, it weakens the immune system more and more making the infected individual much more likely to get other infections called opportunistic infections that are unusual for healthy individuals (AIDS stage) according to CDC. Even though this virus contains on saliva, tear, and urine, but it does not show to be at risk of transmitting the infection among human population since the virus levels on these liquids are very low [1-4]. In HIV infection CD4+T -cells depletion occurs inside the human body with consequent decrease in secretory $\operatorname{IgA}$ production that neutralizes other toxin $[4,5]$. There is no cure or effective vaccine against HIV, however antiretroviral treatment can slow the course of the disease and may lead to a near -normal life expectancy [6-10].

HIV/AIDS transmission in Africa is primarily through heterosexual sex and vertical transmission (mother to-child). $40 \%$ of HIV/AIDS cases result from vertical transmission. High rates of heterosexual transmission in Africa appears to result in part from synergistic (combined action) relationship between HIV and STD. This condition fuel an epidemic among infants and \children of infected mothers. A smaller 
contributor to continuing HIV transmission in Africa is the unintentional use of infected blood transfusions [1, 3, 11]. Sub-Saharan region is the region most affected. In 2017, an estimated $66 \%$ of new HIV infections occurred in this region. South Africa has the largest population of people with HIV of any country in the world, at 7.06 million, as of 2017. In Tanzania, HIV/AIDS was reported to have a prevalence of $4.5 \%$ among Tanzanian adults aged 15-49 in 2017 [12-15].

The whole world is touched by the HIV pandemic, as of 2017, approximately 36.9 million people are infected with HIV globally. In 2018, approximately $43 \%$ are women. There were about 940,000 deaths from AIDS in 2017. The 2015 Global Burden of Disease Study (GBD), in a report published estimated that the global incidence of HIV infection peaked in 1997 at 3.3 million per year. Global incidence fell rapidly from 1997 to 2005, to about 2.6 million per year, but remained stable from 2005 to 2015 [12]. South \& South East Asia (a region with about 2 billion people as of 2010 , over $30 \%$ of the global population) has an estimated 4 million cases ( $12 \%$ of all people infected with HIV), with about 250,000 deaths in 2010. Approximately 2.5 million of these cases are in India, where however the prevalence is only about $0.3 \%$ (somewhat higher than that found in Western and Central Europe or Canada). Prevalence is lowest in East Asia at $0.1 \%$. In 2008, approximately 1.2 million people in the United States had HIV; $20 \%$ did not realize that they were infected. Over the 10 -year period from 1999 to 2008, it resulted in about 17,500 deaths per year. In the United Kingdom, as of 2016, there were approximately 89,400 cases and 428 deaths. In Australia, as of 2017, there were about 27,545 cases. In Canada as of 2016, there were about 63,110 cases $[12,16]$.

The Global HIV prevention coalition was launched in October 2017 to reduce new HIV infection by $75 \%$ by 2020 from 2011 baseline. 25 countries with the highest number of new HIV infections were selected for the Global HIV prevention coalition. Of these, 17 are African countries including Ethiopia [1, 17]. Treatment consists of high active antiretroviral therapy (HARRT) which slows the progression of the disease. The goal of ART is to decrease HIV related morbidity and mortality. In addition, it also decreases the risk of transmission of the virus to sexual partners and mother-to-child transmission. [18].

Mathematical modeling provides us invaluable management tools to understand the status of the spread of the disease and to identify and predict the effectiveness of different control measures. Many mathematical models have been used to examine the consequences of HIV infection at the population level. Such as in 2012 the research work conducted by Abdallah S. Waziri et al. in Tanzania with title 'Mathematical Modelling of HIV/AIDS Dynamics with Treatment and Vertical Transmission', the result of their study show that using treatment measures (ARVs) and control of the rate of vertical transmission have the effect of reducing the transmission of the disease significantly[11]. The research work conducted by Maimunah and Dipo Aldila in Indonesia, published in 2018, with title 'Mathematical model for HIV spreads control program with ART treatment', suggest that
ART treatment as an alternative way to control the spread of HIV [4]. However, the goal of this paper is to propose HIV/AIDS dynamics incorporating treatment and awareness on the proper procedure of ART treatment control strategies in the transmission of HIV virus.

The organization of this paper is as follows: In section 2, we formulate our model for HIV/AIDS infection transmission dynamics. We analyze the positivity and boundedness of the solutions of the dynamical system as basic properties of the model, which are essential in the proofs of stability, existence of disease-free and endemic equilibria as well as their local and global stability and analysis of the sensitivity of the parameters of the reproduction number are treated in section 3. In Section 4, we present the numerical simulation to support the analytical results of the research. The discussion, conclusion and recommendation of the research are present in sections 5 and 6 respectively. In the end, we present acknowledgement of the research in last section.

\section{Formulation of the Model}

In this model of HIV infection, public health intervention strategies are incorporated. Individuals living with HIV/AIDS are grouped according to different stages of infections depending on their viral counts. The basic assumptions in developing this model are as follows: The total human population is closed. The susceptible become HIV infected because of direct contact (free sex), blood transfusion from an infected human or from the use of a syringe from an infected human (Horizontal transmission). This infection is assumed not to lead the birth of infected children. Public health intervention strategies of ART treatment on infected individuals and continuous education campaign on the proper procedure of ART (antiretroviral therapy) treatment to avid the failure of ART, will be taken in to account.

The variables and parameters of the model are defined as follows: The total human population $N(t)$ is divided into five classes: susceptible $S(t), H_{I}(t)$ is HIV infected in acute stage, $H_{2}(t)$ is pre-AIDS patients, AIDS patients $A(t)$ and HIV/AIDS treated class $T_{H}(t)$, such that $N(t)=S(t)+$ $H_{I}(t)+H_{2}(t)+A(t)+T_{H}(t) . \mu$ is the natural mortality rate in all classes at time $t$, for $t \geq 0$. We assumed that there is a positive recruitment rate $\Lambda$ into the susceptible class. Susceptible individuals might be infected by direct contact with infected humans with the probability of infection $\beta$ if they are infected by acute individuals $\left(H_{1}\right), \beta \psi_{2}, \beta \psi_{A}$ and $\beta \psi_{H}$ if they are infected by chronic $\left(H_{2}\right)$, AIDS $(A)$ and treated $\left(T_{H}\right)$ compartment individuals respectively. We assume that more infected humans will limit their capability to do daily life activities; therefore, we have $\beta>\beta \psi_{2}>\beta \psi_{A}$. Since the use of treatment significantly reduces the viral load, treated individuals are less infectious than other groups hence $\beta>\beta \psi_{2}>\beta \psi_{A}>\beta \psi_{H}$. Where $\psi_{2}, \psi_{A}$ and $\psi_{H}$ are modification parameters. Therefore, HIV infected individual is generated following the infection of susceptible individuals at a rate $\phi=\frac{\beta\left(H_{1}+\psi_{2} H_{2}+\psi_{A} A+\psi_{\left.H_{H} T_{H}\right)}\right.}{N}$. HIV infective suffer AIDS-induced mortality at a rate $\gamma$. We also assume that the 
infective $\left(H_{1}\right)$ proceed to join the treated class at a rate $\xi_{1}$. Some individuals join the pre-AIDS class $\left(H_{2}\right)$ with a rate $\xi_{2}$, while others seriously infected individuals without showing symptoms move directly to the full blown AIDS class at a rate $\xi_{3}$. The pre-AIDS individuals in class $\left(H_{2}\right)$ move to treated class $\left(T_{H}\right)$ at a rate $\tau_{1}$ and others move to AIDS class $(A)$ at a rate $\tau_{2}$ to develop full blown AIDS. The full-blown AIDS class $(A)$ individuals move to treated class with a rate $\omega$ to get treatment. Individuals in the treatment class $\left(T_{H}\right)$ may not follow proper procedure of ART treatment due to lack of awareness about the procedure. These individuals lead to the failure of treatment and move to AIDS class $(A)$ by the rate of failure of treatment $\varepsilon$..

These assumptions ar translated to the following Schematic flow diagram and dynamical system:

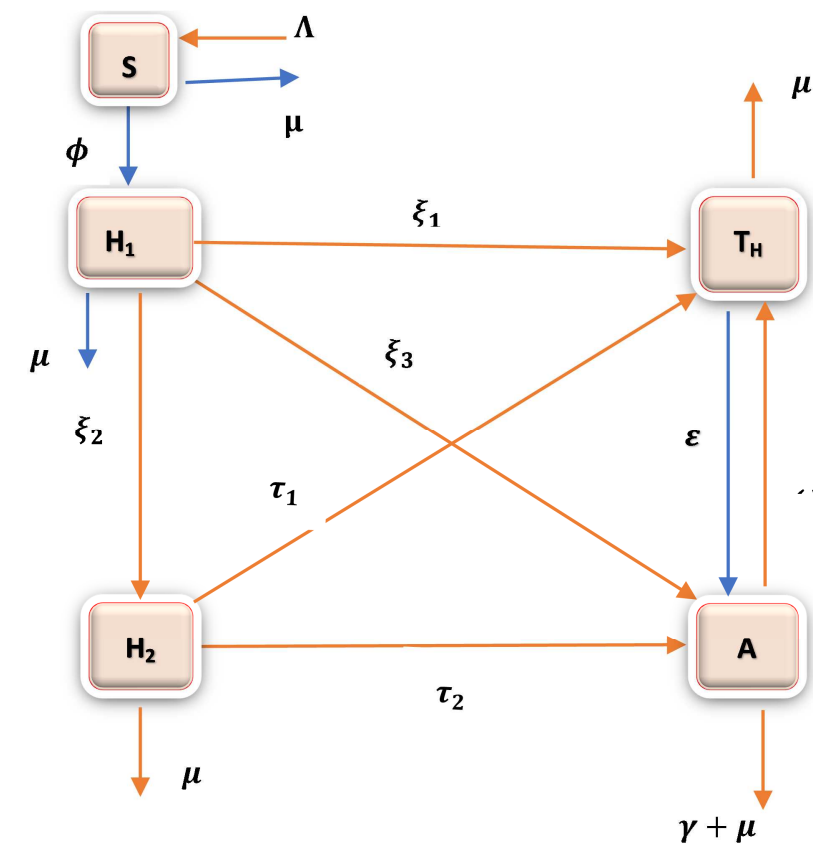

Figure 1. Schematic flow diagram for the model.

$$
\begin{gathered}
\frac{d S}{d t}=\Lambda-\frac{\beta\left(H_{1}+\psi_{2} H_{2}+\psi_{A} A+\psi_{H} T_{H}\right)}{N} S-\mu S \\
\frac{d H_{1}}{d t}=\frac{\beta\left(H_{1}+\psi_{2} H_{2}+\psi_{A} A+\psi_{H} T_{H}\right)}{N} S-\left(\xi_{1}+\xi_{2}+\xi_{3}+\mu\right) H_{1} \\
\frac{d H_{2}}{d t}=\xi_{2} H_{1}-\left(\tau_{1}+\tau_{2}+\mu\right) H_{2} \\
\frac{d A}{d t}=\xi_{3} H_{1}+\tau_{2} H_{2}+\varepsilon T_{H}-(\omega+\gamma+\mu) A \\
\frac{d T_{H}}{d t}=\xi_{1} H_{1}+\tau_{1} H_{2}+\omega A-(\varepsilon+\mu) T_{H}
\end{gathered}
$$

\section{Model Analysis}

\subsection{Positivity of the Solutions}

Theorem 1. The solutions $\left(S(t), H_{1}(t), H_{2}(t), A(t)\right.$ and $T_{H}(t)$ ) of model (1) are nonnegative for all $t>0$ with non-negative initial conditions.
Proof: Since the system of equations (1) represents human populations, all parameters in the model are non-negative and the total human population is finite at time $t>0$, we need to show that, given non-negative initial values

$S(0) \geq 0, H_{1}(0) \geq 0, H_{2}(0) \geq 0, A(0) \geq 0, T_{H}(0) \geq 0$ and, the solutions of the system are non-negative.

Suppose

$$
S(t)+H_{1}(t)+H_{2}(t)+A(t)+T_{H}(t)=N(t) \leq \frac{\Lambda}{\mu}
$$

Let us consider the region

$\Omega=\left\{\left(S, H_{1}, H_{2}, A, T_{H}\right) \in \mathbb{R}^{5}: N \leq \frac{\Lambda}{\mu}\right\}$ for system (1).

From the first equation of the system (1),

$\frac{d S}{d t}=\Lambda-(\phi+\mu) S$, Where $\phi=\frac{\beta\left(H_{1}+\psi_{2} H_{2}+\psi_{A} A+\psi_{H} T_{H}\right)}{N}$

We obtain

$$
\frac{d s}{d t} \leq \Lambda
$$

Therefore, since $S(0) \geq 0$, for $t \rightarrow \infty$,

Which gives $0 \leq S(t) \leq \Lambda t$

From the second equation of the system (1),

$$
\frac{d H_{1}}{d t}=\phi S-\left(\xi_{1}+\xi_{2}+\xi_{3}+\mu\right) H_{1},
$$

We obtain

$$
\frac{d H_{1}}{d t} \leq \phi S
$$

Which gives $H_{1}(t)=e^{-\phi t} \frac{\Lambda}{\mu}\left[e^{\phi t}-1\right] \geq 0$

Therefore, since $H_{1}(0) \geq 0$, for $t \rightarrow \infty$,

$$
0 \leq H_{1}(t) \leq e^{-\phi t} \frac{\Lambda}{\mu}\left[e^{\phi t}-1\right]
$$

From the third equation of the system (1),

$$
\frac{d H_{2}}{d t}=\xi_{2} H_{1}-\left(\tau_{1}+\tau_{2}+\mu\right) H_{2}
$$

We have

$$
\frac{d H_{2}}{d t} \leq \xi_{2} H_{1}
$$

Therefore, since $H_{2}(0) \geq 0$, for $t \rightarrow \infty$,

$$
0 \leq H_{2}(t) \leq e^{-\xi_{2} t} \frac{\Lambda}{\mu}\left[e^{\xi_{2} t}-1\right] \geq 0
$$

From the fourth equation of the system (1),

$$
\frac{d A}{d t}=\xi_{3} H_{1}+\tau_{2} H_{2}+\varepsilon T_{H}-(\omega+\gamma+\mu) A
$$

We get

$$
\begin{gathered}
\frac{d A}{d t} \leq \xi_{3} H_{1}+\tau_{2} H_{2}+\varepsilon T_{H} \\
\Rightarrow A(t)=e^{-x t} x \frac{\Lambda}{\mu} \frac{1}{x}\left[e^{x t}-1\right]=e^{-x t} \frac{\Lambda}{\mu}\left[e^{x t}-1\right] \geq 0
\end{gathered}
$$


Therefore, since $A(0) \geq 0$, for $t \rightarrow \infty$,

$$
0 \leq A(t) \leq e^{-x t} \frac{\Lambda}{\mu}\left[e^{x t}-1\right]
$$

From the fifth equation of the system (1),

$$
\frac{d T_{H}}{d t}=\xi_{1} H_{1}+\tau_{1} H_{2}+\omega A-(\varepsilon+\mu) T_{H},
$$

we obtain

$$
\frac{d T_{H}}{d t} \leq \xi_{1} H_{1}+\tau_{1} H_{2}+\omega A
$$

Therefore, since $T_{H}(0) \geq 0$, for $t \rightarrow \infty$,

$0 \leq T_{H}(t) \leq e^{-y t} \frac{\Lambda}{\mu}\left[e^{y t}-1\right]$, where, $y=\left(\xi_{1}+\tau_{1}+\omega\right)$.

Hence, any solution of system $\left(S(t), H_{1}(t), H_{2}(t), A(t), T_{H}(t)\right) \in \mathbb{R}_{+}^{5}$ for all $t \geq 0$.

\subsection{Boundedness of the Solutions}

Theorem 2.

The Solutions (i.e $S(t), H_{1}(t), H_{2}(t), A(t), T_{H}(t)$ of model (1) are bounded.

Proof:- The total human population in our model is denoted by $N$ and divided in to five subclass which are denoted by $S, H_{1}, H_{2}, A, T_{H}$ from this we have

$$
N(t)=S(t)+H_{I}(t)+H_{2}(t)+A(t)+T_{H}(t)
$$

By differentiating both side with respect to time $t$ we get

$$
\begin{gathered}
\frac{d N}{d t}=\frac{d S}{d t}+\frac{d H_{1}}{d t}+\frac{d H_{2}}{d t}+\frac{d A}{d t}+\frac{d T_{H}}{d t} \\
\Rightarrow \frac{d N}{d t} \leq \Lambda-\mu N .
\end{gathered}
$$

If $\frac{d N}{d t} \geq 0$ then $\Lambda-\mu N \geq 0$. Thus $N(t) \leq \frac{\Lambda}{\mu}$ for $N(0) \leq \frac{\Lambda}{\mu^{\prime}}$ where at $t=0, N(0)$ is initial population. Therefore $\lim _{t \rightarrow \infty} \sup N(t) \leq \frac{\Lambda}{\mu}$. This shows that $N(t)$ is bounded above and increasing. Since $N(t)$ is bounded above, each other state variable of human population $S(t), H_{1}(t), H_{2}(t), A(t)$, or $T_{H}(t)$ is bounded above.

To show that the state variables of human population are bounded below, we need to show the boundedness of each state variable below for $N(0) \geq \frac{\Lambda}{\mu}$.

From the first equation of the system (1), we have

$$
\frac{d S}{d t} \leq \Lambda-\phi S
$$

Thus, $S(t)$ is bounded below and decreasing. Therefore,

$$
\lim _{t \rightarrow \infty} \inf \mathrm{S}(\mathrm{t}) \geq \frac{\Lambda}{\phi}
$$

From the second equation of the system (1),

$$
\frac{d H_{1}}{d t}=\phi S-\left(\xi_{1}+\xi_{2}+\xi_{3}+\mu\right) H_{1}
$$

If $H_{I}(t)$ is decreasing with time $t>0, \frac{d H_{I}}{d t} \leq 0$

$$
\Rightarrow H_{1}(t) \geq \frac{\Lambda}{\mu}\left(\frac{\phi}{\phi+\xi_{1}+\xi_{2}+\xi_{3}+\mu}\right)
$$

Which gives,

$$
\lim _{t \rightarrow \infty} \inf H_{1}(t) \geq \frac{\Lambda}{\mu}\left(\frac{\phi}{\phi+\xi_{1}+\xi_{2}+\xi_{3}+\mu}\right)
$$

This shows that $H_{I}(t)$ is bounded below. From the third equation of the system (1),

$$
\frac{d H_{2}}{d t}=\xi_{2} H_{1}-\left(\tau_{1}+\tau_{2}+\mu\right) H_{2}
$$

If $H_{2}(t)$ is decreasing with time $t>0, \frac{d H_{2}}{d t} \leq 0$ Therefore,

$$
\lim _{t \rightarrow \infty} \inf H_{2}(t) \geq \frac{\Lambda}{\mu}\left(\frac{\xi_{2}}{\xi_{2}+\tau_{1}+\tau_{2}+\mu}\right)
$$

This shows that $H_{2}(t)$ is bounded below. From the fourth equation of the system (1)

$$
\frac{d A}{d t}=\xi_{3} H_{1}+\tau_{2} H_{2}+\varepsilon T_{H}-(\omega+\gamma+\mu) A
$$

If $A(t)$ is decreasing with time $t>0, \frac{d A}{d t} \leq 0$ that is $\mathrm{A}(\mathrm{t}) \geq \frac{\Lambda}{\mu}\left(\frac{u}{v}\right)$,

Where $u=\left(\xi_{3}+\tau_{2}+\varepsilon\right)$ and $v=\left(\xi_{3}+\tau_{2}+\varepsilon+\omega+\right.$ $\gamma+\mu)$.

$$
\text { Therefore, } \lim _{t \rightarrow \infty} \text { inf } \mathrm{A}(\mathrm{t}) \geq \frac{\Lambda}{\mu}\left(\frac{u}{v}\right)
$$

This shows that $A(t)$ is bounded below. From the fifth equation of the system (1)

$$
\frac{d T_{H}}{d t}=\xi_{1} H_{1}+\tau_{1} H_{2}+\omega A-(\varepsilon+\mu) T_{H}
$$

If $T_{H}(t)$ is decreasing with time $t>0, \frac{d T_{H}}{d t} \leq 0$

Therefore, $\lim _{t \rightarrow \infty}$ inf $T_{H}(t) \geq \frac{\Lambda}{\mu}\left(\frac{\xi_{1}+\tau_{1}+\omega}{\xi_{1}+\tau_{1}+\omega+\varepsilon+\mu}\right)$

This shows that $T_{H}(t)$ is bounded below.

Hence, all solutions of the system (1) are bounded. Theorem 3. The region

$\Omega=\left\{\left(S, H_{1}, H_{2}, A, T_{H}\right) \in \mathbb{R}_{+}^{5}: N \leq \frac{\Lambda}{\mu}\right\} \quad$ is $\quad$ positively invariant for the model (1) with non-negative initial conditions in $\mathbb{R}_{+}^{5}$.

Proof: To proof the positive invariance of $\Omega$ (i.e., all solutions in $\Omega$ remain in $\Omega$ for all $t$ ).

$$
\begin{gathered}
\text { Let } \frac{d N}{d t}=\Lambda-\mu N \\
\Rightarrow N(t)=\frac{\Lambda}{\mu}-\frac{\Lambda}{\mu} e^{-\mu t} \leq \frac{\Lambda}{\mu}-N(0) e^{-\mu t}, \text { for } N(0) \leq \frac{\Lambda}{\mu} \\
\Rightarrow N(t) \leq \frac{\Lambda}{\mu}-N(0) e^{-\mu t} \\
\text { If } \frac{d N}{d t} \geq 0 \text { then } \Lambda-\mu N \geq 0 \text { and } \frac{d N}{d t} \geq 0 \Rightarrow N(t) \leq \frac{\Lambda}{\mu}
\end{gathered}
$$


Specifically, if $\frac{d N}{d t} \geq 0$ then $N(0) \leq \frac{\Lambda}{\mu}$. Thus $N(t) \leq \frac{\Lambda}{\mu}$ and this shows that $N(t)$ is increasing and bounded above by $\frac{\Lambda}{\mu}$ and if $\Lambda-\mu N<0$ then $\frac{d N}{d t}<0$.

Thus $N(t)>\frac{\Lambda}{\mu} \Rightarrow \frac{d N}{d t}<0$. In particular, if $N(0)>\frac{\Lambda}{\mu^{\prime}}$ then $\frac{d N}{d t}<0$ and this shows that $N(t)$ is decreasing and bounded below by 0 because of positivity. Thus, $\Omega$ is positive-invariant and attracting. Therefore, every solution of the dynamical systems with initial conditions in $\Omega$ remains in $\Omega$ for $t>0$. Hence, it is sufficient to consider the dynamics for the system (1) in $\Omega$ and thus, in the region, the model the system (1) is epidemiologically and mathematically well posed.

Corollary. Let $\Omega=\left\{\left(S, H_{1}, H_{2}, A, T_{H}\right) \in \mathbb{R}_{+}^{5}: N \leq \frac{\Lambda}{\mu}\right\}$, then the region is invariant and attracting for system (1). The feasible region is $\Omega=\left\{\left(S, H_{1}, H_{2}, A, T_{H}\right) \in \mathbb{R}_{+}^{5}: N \leq \frac{\Lambda}{\mu}\right\}$

\subsection{Disease -free Equilibrium Point}

The disease -free equilibrium point of the system (1) can be obtained by setting HIV related variables and parameters to zero

$$
H_{1}=H_{2}=A=T_{H}=\phi=0
$$

then we have

$\Lambda-\mu S=0$. Therefor the infection-free equilibrium point of the HIV/AIDS model is

$$
E_{o h}=\left(S_{0}, H_{1_{0}}, H_{2_{0}}, A_{0}, T_{H_{0}}\right)=\left(\frac{\Lambda}{\mu}, 0,0,0,0\right)
$$

\subsection{Reproduction Number}

Theorem 4. The effective reproduction number $\left(R_{h}\right)$ of HIV/AIDS model (1) is given by

$$
\mathrm{R}_{\mathrm{h}}=\frac{\beta \Lambda}{N \mu}\left(\frac{-\varepsilon \omega c_{2}+c_{2} c_{3} c_{4}+\psi_{2}\left(-\varepsilon \omega \xi_{2}+c_{3} c_{4} \xi_{2}\right)+\psi_{A}\left(c_{2} c_{4} \xi_{3}+\varepsilon c_{2} \xi_{1}+\varepsilon \xi_{2} \tau_{1}+c_{4} \xi_{2} \tau_{2}\right)+\psi_{H}\left(\omega c_{2} \xi_{3}+c_{2} c_{3} \xi_{1}+c_{3} \xi_{2} \tau_{1}+\omega \xi_{2} \tau_{2}\right)}{\left(-\varepsilon \omega c_{1} c_{2}+c_{1} c_{2} c_{3} c_{4}\right)}\right)
$$

Proof: In order to compute $R_{h}$, it is important to distinguish new infections from all other changes in the host population. We apply the next generation approach in Diekmann et al. 1990 [23].

$$
\frac{d x_{i}}{d t}=f_{i}(t)=\mathcal{F}_{i}(t)-v_{i}(t)
$$

where $i=1, \ldots, 5$

$$
v_{i}(t)=V_{i}^{-}(t)-V_{i}^{+}(t)
$$

and the matrices $\mathcal{F}(t), V^{+}(t)$ and $V^{-}(t)$ associated with model (1) are given by

$$
\begin{gathered}
\mathcal{F}(t)=\left[\begin{array}{c}
\frac{\beta\left(H_{1}+\psi_{2} H_{2}+\psi_{A} A+\psi_{H} T_{H}\right)}{N} S \\
0 \\
0 \\
0 \\
0 \\
0
\end{array}\right], \\
V^{+}(t)=\left[\begin{array}{c}
\xi_{2} H_{1}(t) \\
\xi_{3} H_{1}(t)+\tau_{2} H_{2}+\varepsilon T_{H} \\
\xi_{1} H_{1}+\tau_{1} H_{2}+\omega A \\
\Lambda
\end{array}\right] \text { and } V^{-}(t)=\left[\begin{array}{c}
c_{1} H_{1}(t) \\
c_{2} H_{2}(t) \\
c_{3} A(t) \\
c_{4} T_{H}(t) \\
c_{5} S(t)
\end{array}\right]
\end{gathered}
$$

Where, $c_{1}=\xi_{1}+\xi_{2}+\xi_{3}+\mu, c_{2}=\tau_{1}+\tau_{2}+\mu$,

$$
c_{3}=\omega+\gamma+\mu, c_{4}=\varepsilon+\mu, c_{5}=\phi+\mu
$$

Let $v_{i}(t)=V_{i}^{-}(t)-V_{i}^{+}(t)$

Then, we have

$$
\left[\frac{d H_{1}(t)}{d t} \frac{d H_{2}(t)}{d t} \frac{d A(t)}{d t} \frac{d T_{H}(t)}{d t} \frac{d S(t)}{d t}\right]^{T}=\mathcal{F}(t)-v(t)
$$

The Jacobian matrices of $F(t)$ and $V(t)$ for
$\mathcal{F}(t)$ and $v(t)$ respectively are $4 \times 4$ matrices as there are four infected classes

$$
F=\frac{\partial \mathcal{F}}{\partial x_{i}}=\left[\begin{array}{cccc}
\frac{\beta}{N} S(t) & \frac{\beta \psi_{2}}{N} S(t) & \frac{\beta \psi_{A}}{N} S(t) & \frac{\beta \psi_{H}}{N} S(t) \\
0 & 0 & 0 & 0 \\
0 & 0 & 0 & 0 \\
0 & 0 & 0 & 0
\end{array}\right]
$$

and $\quad V=\frac{\partial v}{\partial x_{i}}=\left[\begin{array}{cccc}c_{1} & 0 & 0 & 0 \\ -\xi_{2} & c_{2} & 0 & 0 \\ -\xi_{3} & -\tau_{2} & c_{3} & -\varepsilon \\ -\xi_{1} & -\tau_{1} & -\omega & c_{4}\end{array}\right]$, where $x_{i}=$ $\left(H_{1}, H_{2}, A, T_{H}\right)$.

At the infection-free equilibrium pint $E_{o h}, F$ and $V$ at $E_{o h} F_{0}$ and $V_{0}$ respectively are:

$$
\begin{gathered}
F_{0}=\left[\begin{array}{cccc}
\frac{\beta \Lambda}{N \mu} & \frac{\beta \psi_{2} \Lambda}{N \mu} & \frac{\beta \psi_{A} \Lambda}{N \mu} & \frac{\beta \psi_{H} \Lambda}{N \mu} \\
0 & 0 & 0 & 0 \\
0 & 0 & 0 & 0 \\
0 & 0 & 0 & 0
\end{array}\right] \text { and } \\
V_{0}=\left[\begin{array}{cccc}
c_{1} & 0 & 0 & 0 \\
-\xi_{2} & c_{2} & 0 & 0 \\
-\xi_{3} & -\tau_{2} & c_{3} & -\varepsilon \\
-\xi_{1} & -\tau_{1} & -\omega & c_{4}
\end{array}\right]
\end{gathered}
$$

Thus $. R_{h}=\rho\left(F_{0} \mathrm{~V}_{0}^{-1}\right)$ Where $\rho\left(F_{0} \mathrm{~V}_{0}^{-1}\right)$ denotes the spectral radius of a matrix $F_{0} \mathrm{~V}_{0}^{-1}$ (that is, the eigenvalue with the highest magnitude). To find the eigen values of $F_{0} \mathrm{~V}_{0}^{-1}$, we consider $\operatorname{det}\left(F_{0} \mathrm{~V}_{0}^{-1}-\lambda I\right)=0$, where $I i$ s the identity matrix.

Thus, $\Rightarrow \lambda=0$ or $\lambda=\frac{\beta n_{1} \Lambda}{D N \mu}+\frac{\beta \psi_{2} n_{2} \Lambda}{D N \mu}+\frac{\beta \psi_{A} n_{4} \Lambda}{D N \mu}+\frac{\beta \psi_{H} n_{8} \Lambda}{D N \mu}$

$$
\text { Thus, } R_{h}=\frac{\beta n_{1} \Lambda+\beta \psi_{2} n_{2} \Lambda+\beta \psi_{A} n_{4} \Lambda+\beta \psi_{H} n_{8} \Lambda}{D N \mu}
$$

$$
\mathrm{R}_{\mathrm{h}}=\frac{\beta \Lambda}{N \mu}\left(\frac{-\varepsilon \omega c_{2}+c_{2} c_{3} c_{4}+\psi_{2}\left(-\varepsilon \omega \xi_{2}+c_{3} c_{4} \xi_{2}\right)+\psi_{A}\left(c_{2} c_{4} \xi_{3}+\varepsilon c_{2} \xi_{1}+\varepsilon \xi_{2} \tau_{1}+c_{4} \xi_{2} \tau_{2}\right)+\psi_{H}\left(\omega c_{2} \xi_{3}+c_{2} c_{3} \xi_{1}+c_{3} \xi_{2} \tau_{1}+\omega \xi_{2} \tau_{2}\right)}{\left(-\varepsilon \omega c_{1} c_{2}+c_{1} c_{2} c_{3} c_{4}\right)}\right)
$$


Where, $c_{1}=\xi_{1}+\xi_{2}+\xi_{3}+\mu, c_{2}=\tau_{1}+\tau_{2}+\mu$,

$$
c_{3}=\omega+\gamma+\mu, c_{4}=\varepsilon+\mu
$$

Which represent the average number of new secondary cases generated by a single HIV infected individual during his/her entire infectious period in a completely susceptible population, in the presence of HIV intervention strategy [19-21].

Theorem 5. The basic reproduction number $\left(\mathrm{R}_{0 h}\right)$ of HIV transmission model (1) is given by

$$
\mathrm{R}_{0 \mathrm{~h}}=\frac{\beta}{\xi_{2}+\xi_{3}+\mu}\left(1+\frac{\psi_{2} \xi_{2}}{\tau_{2}+\mu}+\frac{\psi_{A} \xi_{3}}{\gamma+\mu}+\frac{\psi_{A} \xi_{2} \tau_{2}}{\left(\tau_{2}+\mu\right)(\gamma+\mu)}\right)
$$

Proof: We apply the next generation approach in Diekmann et al. [23] by setting $\xi_{1}=\tau_{1}=\omega=T_{H}=0$ in mode (1):

$$
\frac{d x_{i}}{d t}=f_{i}(t)=\mathcal{F}_{i}(t)-v_{i}(t)
$$

Where, $i=1,2,3,4$, as there are three classes and

$$
x_{i}=\left(H_{1}, H_{2}, A, S\right)
$$

$v_{i}(t)=V_{i}^{-}(t)-V_{i}^{+}(t) \quad$ and the matrices $\mathcal{F}(t), V^{+}(t)$ and $V^{-}(t)$ are given by

$$
\begin{gathered}
\mathcal{F}(t)=\left[\begin{array}{c}
\frac{\beta\left(H_{1}+\psi_{2} H_{2}+\psi_{A} A\right)}{N} S \\
0 \\
0 \\
0
\end{array}\right], \\
V^{+}(t)=\left[\begin{array}{c}
0 \\
\xi_{2} H_{1}(t) \\
\xi_{3} H_{1}+\tau_{2} H_{2} \\
\Lambda
\end{array}\right] \text { and } V^{-}(t)=\left[\begin{array}{c}
c_{7} H_{1}(t) \\
c_{8} H_{2}(t) \\
c_{9} A(t) \\
c_{10} S(t)
\end{array}\right]
\end{gathered}
$$

Where, $c_{7}=\xi_{2}+\xi_{3}+\mu, c_{8}=\tau_{2}+\mu, c_{9}=\gamma+\mu$,

$$
c_{10}=\phi+\mu
$$

Let, $v_{i}(t)=V_{i}^{-}(t)-V_{i}^{+}(t)$

Then, we have

$$
\left[\frac{d H_{1}(t)}{d t} \frac{d H_{2}(t)}{d t} \frac{d A(t)}{d t} \frac{d S(t)}{d t}\right]^{T}=\mathcal{F}(t)-v(t)
$$

The Jacobian matrices of $F(t)$ and $V(t)$ for $\mathcal{F}(t)$ and $v(t)$ respectively are $3 \times 3$ matrices as there are five infected classes

$$
\begin{aligned}
& F=\frac{\partial \mathcal{F}}{\partial x_{i}}=\left[\begin{array}{ccc}
\frac{\beta}{N} S(t) & \frac{\beta \psi_{2}}{N} S(t) & \frac{\beta \psi_{A}}{N} S(t) \\
0 & 0 & 0 \\
0 & 0 & 0
\end{array}\right] \text { and } V=\frac{\partial v}{\partial x_{i}}= \\
& b_{2}=\left(-\varepsilon \omega-\beta c_{4}+c_{1} c_{4}+c_{3}\left(-\beta+c_{1}+c_{4}\right)+c_{2}\left(-\beta+c_{1}+c_{3}+c_{4}\right)-\beta \xi_{2} \psi_{2}-\beta \xi_{3} \psi_{A}-\beta \xi_{1} \psi_{H}\right) \\
& b_{1}=\left(c_{1}\left(-\varepsilon \omega+c_{3} c_{4}+c_{2}\left(c_{3}+c_{4}\right)\right)-c_{2}\left(\varepsilon \omega+c_{3}\left(\beta-c_{4}\right)+\beta c_{4}+\beta \xi_{3} \psi_{A}+\beta \xi_{1} \psi_{H}\right)+\beta\left(\varepsilon \omega-\varepsilon \xi_{1} \psi_{A}-\xi_{2} \tau_{2} \psi_{A}\right.\right. \\
& \left.\left.-c_{4}\left(\xi_{2} \psi_{2}+\xi_{3} \psi_{A}\right)-\omega \xi_{3} \psi_{H}-\xi_{2} \tau_{1} \psi_{H}-c_{3}\left(c_{4}+\xi_{2} \psi_{2}+\xi_{1} \psi_{H}\right)\right)\right)
\end{aligned}
$$

At the infection-free equilibrium pint $E_{o h} S=N, F$ and $V$ at $E_{o h}$ are $F_{0}$ and $V_{0}$ respectively:

$$
F_{0}=\left[\begin{array}{ccc}
\beta & \beta \psi_{2} & \beta \psi_{A} \\
0 & 0 & 0 \\
0 & 0 & 0
\end{array}\right] \text { and } V_{0}=\left[\begin{array}{ccc}
c_{7} & 0 & 0 \\
-\xi_{2} & c_{8} & 0 \\
-\xi_{3} & -\tau_{2} & c_{9}
\end{array}\right] \text {, }
$$

Thus $\cdot R_{o h}=\rho\left(F_{0} \mathrm{~V}_{0}^{-1}\right)$, where $\rho\left(F_{0} \mathrm{~V}_{0}^{-1}\right)$ denotes the spectral radius of a matrix $F_{0} \mathrm{~V}_{0}^{-1}$ (i.e, the eigenvalue with the highest magnitude). To find the eigen values of $F_{0} \mathrm{~V}_{0}^{-1}$, we set $\operatorname{det}\left(F_{0} \mathrm{~V}_{0}^{-1}-\lambda I\right)=0$, where $I$ is the identity matrix.

Thus,

$$
\mathrm{R}_{0 \mathrm{~h}}=\frac{\beta}{\xi_{2}+\xi_{3}+\mu}\left(1+\frac{\psi_{2} \xi_{2}}{\tau_{2}+\mu}+\frac{\psi_{A} \xi_{3}}{\gamma+\mu}+\frac{\psi_{A} \xi_{2} \tau_{2}}{\left(\tau_{2}+\mu\right)(\gamma+\mu)}\right)
$$

\subsection{Local Stability of the Disease-free Steady State}

Theorem 6. The infection-free equilibrium $\left(E_{o h}\right)$ of model (1) is locally asymptotically stable, if $R_{h}<1$ and unstable, if $\mathrm{R}_{\mathrm{h}}>1$.

Proof: The Jacobian matrix of the system (1) at the disease free equilibrium point $E_{0 h}$ is

$$
J\left(E_{0 h}\right)=\left[\begin{array}{ccccc}
-\mu & -\beta & -\beta \psi_{2} & -\beta \psi_{A} & -\beta \psi_{H} \\
0 & \beta-c_{1} & \beta \psi_{2} & \beta \psi_{A} & \beta \psi_{H} \\
0 & \xi_{2} & -c_{2} & 0 & 0 \\
0 & \xi_{3} & \tau_{2} & -c_{3} & \varepsilon \\
0 & \xi_{1} & \tau_{1} & \omega & -c_{4}
\end{array}\right],
$$

as $S=N$ at $E_{O h}$, Where, $c_{1}=\left(\xi_{1}+\xi_{2}+\xi_{3}+\mu\right), c_{2}=$ $\left(\tau_{1}+\tau_{2}+\mu\right), c_{3}=(\omega+\gamma+\mu), c_{4}=(\varepsilon+\mu)$

The characteristic polynomial of the matrix $J\left(E_{0 h}\right)$ is given by $p_{5}(\lambda)=\operatorname{det}\left(J\left(E_{0 h}\right)-\lambda \mathrm{I}\right)$ and in order to find the roots of the polynomial $p$, we set

$$
\begin{gathered}
p_{5}(\lambda)=\left|\begin{array}{ccccc}
-\mu-\lambda & -\beta & -\beta \psi_{2} & -\beta \psi_{A} & -\beta \psi_{H} \\
0 & \beta-c_{1}-\lambda & \beta \psi_{2} & \beta \psi_{A} & \beta \psi_{H} \\
0 & \xi_{2} & -c_{2}-\lambda & 0 & 0 \\
0 & \xi_{3} & \tau_{2} & -c_{3}-\lambda & \varepsilon \\
0 & \xi_{1} & \tau_{1} & \omega & -c_{4}-\lambda
\end{array}\right|=0 \\
p_{5}(\lambda)=(-\mu-\lambda)\left[b_{4} \lambda^{4}+b_{3} \lambda^{3}+b_{2} \lambda^{2}+b_{1} \lambda^{1}+b_{0}\right]=0
\end{gathered}
$$

$\Rightarrow \lambda=-\mu<0$ or $b_{4} \lambda^{4}+b_{3} \lambda^{3}+b_{2} \lambda^{2}+b_{1} \lambda^{1}+b_{0}=0$

Where, $b_{4}=1, b_{3}=\left(-\beta+c_{1}+c_{2}+c_{3}+c_{4}\right)$ 


$$
\begin{gathered}
b_{0}=\beta \xi_{2}\left(\left(\varepsilon \omega-c_{3} c_{4}\right) \psi_{2}-\tau_{2}\left(c_{4} \psi_{A}+\omega \psi_{H}\right)-\tau_{1}\left(\varepsilon \psi_{A}+c_{3} \psi_{H}\right)\right) \\
+c_{2}\left(c_{1}\left(-\varepsilon \omega+c_{3} c_{4}\right)+\beta\left(\varepsilon \omega-\varepsilon \xi_{1} \psi_{A}-c_{4} \xi_{3} \psi_{A}-\omega \xi_{3} \psi_{H}-c_{3}\left(c_{4}+\xi_{1} \psi_{H}\right)\right)\right)
\end{gathered}
$$

The disease free steady state is locally asymptotically stable, if the necessary and sufficient conditions that all the roots of the polynomial in (29) have negatives real parts are all the polynomial coefficients must have the same sign (all positive in this case), nonzero. In addition, the first column containing the term $b_{4}$ of the Routh's array should be positive by Routh-Hurwitz stability criterion.

The biological interpretation of locally asymptotically stable of the disease-free equilibrium point is that, the existence of small number of infectious individuals will not be the cause of the outbreak of the disease unless $R_{h}>1$.

$$
V\left(S, H_{1}, H_{2}, A, T_{H}\right)=a_{1} H_{1}+a_{2} H_{2}+a_{3} A+a_{4} T_{H}
$$

where

$$
\text { Let } a_{1}=c_{2}\left(c_{3} c_{4}-\varepsilon \omega\right)+\psi_{2}\left(-\varepsilon \omega \xi_{2}+c_{3} c_{4} \xi_{2}\right)+\underset{A}{\psi_{A}\left(c_{2} c_{4} \xi_{3}+\varepsilon c_{2} \xi_{1}+\varepsilon \xi_{2} \tau_{1}+c_{4} \xi_{2} \tau_{2}\right)+\psi_{H}\left(\omega c_{2} \xi_{3}+c_{2} c_{3} \xi_{1}+c_{3} \xi_{2} \tau_{1}+\right.}+
$$

$a_{2}=c_{1}\left[\psi_{2}\left(-\varepsilon \omega+c_{3} c_{4}\right)+\psi_{A}\left(\varepsilon \tau_{1}+c_{4} \tau_{2}\right)+\psi_{H}\left(c_{3} \tau_{1}+\omega \tau_{2}\right)\right], a_{3}=c_{1}\left[\psi_{A} c_{2} c_{4}+\psi_{H} \omega c_{2}\right]$ and

$a_{4}=c_{1}\left[\psi_{A} \varepsilon c_{2}+\psi_{H} c_{2} c_{3}\right]$. Since $a_{1}, a_{2}, a_{3}$ and $c_{4}$ are all non negative and also all state variables are positive, $V\left(S, H_{1}, H_{2}, A, T_{H}\right) \geq 0$ and

$V\left(S, H_{1}, H_{2}, A, T_{H}\right)=V\left(\frac{\Lambda}{\mu}, 0,0,0,0\right)=0$ is the minimum value.

$\frac{\partial V}{\partial H_{1}}=a_{1}, \frac{\partial V}{\partial H_{2}}=a_{2}, \frac{\partial V}{\partial A}=a_{3}$ and $\frac{\partial V}{\partial T_{H}}=a_{4}$ are all constants and thus continuous partial derivatives.

$$
\begin{gathered}
\frac{d V}{d t}=\frac{\partial V}{\partial H_{1}} \frac{d H_{1}}{d t}+\frac{\partial V}{\partial H_{2}} \frac{d H_{2}}{d t}+\frac{\partial V}{\partial A} \frac{d A}{d t}+\frac{\partial V}{\partial T_{H}} \frac{d T_{H}}{d t}= \\
=\frac{\phi N c_{1}\left[-\varepsilon \omega c_{2}+c_{2} c_{3} c_{4}\right]}{\beta}\left[\frac{a_{1} \phi S \beta}{\phi N c_{1}\left[-\varepsilon \omega c_{2}+c_{2} c_{3} c_{4}\right]}-1\right]=\frac{\phi N c_{1}\left[-\varepsilon \omega c_{2}+c_{2} c_{3} c_{4}\right]}{\beta}\left[\frac{a_{1} S \beta}{N c_{1}\left[-\varepsilon \omega c_{2}+c_{2} c_{3} c_{4}\right]}-1\right] \\
\leq \frac{\phi N c_{1}\left[-\varepsilon \omega c_{2}+c_{2} c_{3} c_{4}\right]}{\beta}\left[\frac{a_{1} \beta}{c_{1}\left[-\varepsilon \omega c_{2}+c_{2} c_{3} c_{4}\right]}-1\right], \text { since } S \leq N=\frac{\phi N c_{1}\left[-\varepsilon \omega c_{2}+c_{2} c_{3} c_{4}\right]}{\beta}\left[R_{h}-1\right] \leq 0
\end{gathered}
$$

when $R_{h} \leq 1$. Since all parameters are non-negative $\frac{d V}{d t} \leq 0$, if $R_{h} \leq 1$ and $\frac{d V}{d t}=0$ holds if and only if $H_{1}=H_{2}=A=$ $T_{H}=0$

The largest compact invariant subsets in $\left\{\left(S, H_{1}, H_{2}, A, T_{H}\right)\right\}$ is the singleton set $\left\{\mathrm{E}_{0 \mathrm{~h}}\right\}$.

Therefore, by Lasalle's invariant principle (Lasalle 1976) $E_{0 h}$ is global attractor whenever $R_{h}<1$ (i.e every solution of the model approaches to $E_{0 h}$ as $t \rightarrow \infty$ with initial conditions in $\Omega$ )

\subsection{Endemic Equilibrium Point}

Theorem 8 . The endemic equilibrium point of the system (1) exist, if $R_{h}>1$.

Proof: The endemic equilibrium point

$E_{h}=\left(S^{*}, H_{1}^{*}, H_{2}^{*}, A, T_{H}^{*}\right)$ of the system (1) can be obtained by setting $\frac{d S}{d t}=\frac{d H_{1}}{d t}=\frac{d H_{2}}{d t}=\frac{d A}{d t}=\frac{d T_{H}}{d t}=0$

Therefore,

$$
E_{h}=\left(S^{*}, H_{1}^{*}, H_{2}^{*}, A^{*}, T_{H}^{*}\right)=\left(\frac{\Lambda}{\phi^{*}+\mu}, \frac{\phi^{*} \Lambda}{c_{1}\left(\phi^{*}+\mu\right)}, \frac{\xi_{2} \phi^{*} \Lambda}{c_{1} c_{2}\left(\phi^{*}+\mu\right)}, \frac{c_{3} P}{Q}, \frac{c_{3} R}{Q},\right)
$$

Where,

$$
\begin{gathered}
P=\left((\varepsilon+\mu) c_{2} \xi_{3} \phi^{*} \Lambda+\left((\varepsilon+\mu) \xi_{2} \tau_{2} \phi^{*} \Lambda\right)+\varepsilon c_{2} \xi_{1} \phi^{*} \Lambda+\varepsilon \tau_{1} \xi_{2} \phi^{*} \Lambda\right. \\
Q=c_{1} c_{2} c_{3}\left(\phi^{*}+\mu\right)\left(c_{3}(\varepsilon+\mu)-\omega \varepsilon\right) \\
R=c_{2} c_{3} \xi_{1} \phi^{*} \Lambda+c_{3} \tau_{1} \xi_{2} \phi^{*} \Lambda+\omega \phi^{*} \Lambda c_{2} \xi_{3}+\omega \phi^{*} \Lambda \xi_{2} \tau_{2}
\end{gathered}
$$

\section{From,}

$$
\phi^{*}=\frac{\beta\left(H_{1}^{*}+\psi_{2} H_{2}^{*}+\psi_{A} A^{*}+\psi_{H} T_{H}^{*}\right)}{N^{*}}
$$

$E_{h}=\left(S^{*}, H_{1}^{*}, H_{2}^{*}, A^{*}, T_{H}^{*}\right)$, we have

$\phi^{*}=-\mu\left(1-\frac{\Lambda}{\mu N^{*}} R_{h}\right)$, but at disease free equilibrium and point $S^{*}=N^{*}$, thus, $\frac{\Lambda}{\mu N^{*}}=1$

$$
\Rightarrow \phi^{*}=-\mu\left(1-R_{h}\right)
$$

We define $\phi^{*}=\frac{\beta\left(H_{1}^{*}+\psi_{2} H_{2}^{*}+\psi_{A} A^{*}+\psi_{H} T_{H}^{*}\right)}{N^{*}}$ is the rate of HIV 
infection and the disease persist in the community if $\phi^{*}>0$, that is endemic equilibrium point exist for $\mathrm{R}_{\mathrm{h}}>1$.

Where, $\quad c_{1}=\left(\xi_{1}+\xi_{2}+\xi_{3}+\mu\right), \quad c_{2}=\left(\tau_{1}+\tau_{2}+\mu\right)$, $c_{3}=(\omega+\gamma+\mu), c_{4}=(\varepsilon+\mu)$

$$
\begin{gathered}
P_{1}=(\varepsilon+\mu) c_{2} \xi_{3}+\left((\varepsilon+\mu) \xi_{2} \tau_{2}\right)+\varepsilon c_{2} \xi_{1}+\varepsilon \tau_{1} \xi_{2} \\
R_{1}=c_{2} c_{3} \xi_{1}+c_{3} \tau_{1} \xi_{2}+\left(\omega c_{2} \xi_{3}+\omega \xi_{2} \tau_{2}\right)
\end{gathered}
$$

$$
J\left(E_{h}\right)=\left[\begin{array}{ccccc}
-g-\mu & -l & -m & -n & -r \\
g & l-\left(\xi_{1}+\xi_{2}+\mu\right) & m & n & r \\
0 & \xi_{2} & -\left(\tau_{1}+\tau_{2}+\mu\right) & 0 & 0 \\
0 & \xi_{3} & \tau_{2} & -(\omega+\gamma+\mu) & \varepsilon \\
0 & \xi_{1} & \tau_{1} & \omega & -(\varepsilon+\mu)
\end{array}\right]
$$

Where, $g=\frac{\beta\left(H_{1}^{*}+\psi_{2} H_{2}^{*}+\psi_{A} A+\psi_{H} T_{H}^{*}\right)}{N^{*}}, l=\frac{\beta S^{*}}{N^{*}}, m=\frac{\beta \psi_{2} S^{*}}{N^{*}}, n=\frac{\beta \psi_{A} S^{*}}{N^{*}}$ and $r=\frac{\beta \psi_{H} S^{*}}{N^{*}}$

Let $c_{1}=\left(\xi_{1}+\xi_{2}+\xi_{3}+\mu\right), c_{2}=\left(\tau_{1}+\tau_{2}+\mu\right), c_{3}=(\omega+\gamma+\mu), c_{4}=(\varepsilon+\mu)$

The characteristic polynomial of the matrix $J\left(E_{h}\right)$ is given by $\mathrm{h}_{5}(\varpi)=\operatorname{det}\left(J\left(E_{h}\right)-\varpi \mathrm{T}\right)$, where $T$ is identity matrix and in order to find the roots of the polynomial $h_{5}(\varpi)$, we set

$$
=\left|\begin{array}{ccccc}
f-\varpi & -l & -m & -n & -r \\
g & q-\varpi & m & n & r \\
0 & \xi_{2} & -c_{2}-\varpi & 0 & 0 \\
0 & \xi_{3} & \tau_{2} & -c_{3}-\varpi & \varepsilon \\
0 & \xi_{1} & \tau_{1} & \omega & -c_{4}-\varpi
\end{array}\right|=0
$$

Where, $f=-g-\mu=-\frac{\beta\left(H_{1}^{*}+\psi_{2} H_{2}^{*}+\psi_{A} A+\psi_{H} T_{H}^{*}\right)}{N^{*}}-\mu$,

$$
\begin{gathered}
q=l-c_{1}=\frac{\beta S^{*}}{N^{*}}-\left(\xi_{1}+\xi_{2}+\xi_{3}+\mu\right),=\frac{\beta \psi_{2} S^{*}}{N^{*}}, n=\frac{\beta \psi_{A} S^{*}}{N^{*}} \text { and } r=\frac{\beta \psi_{H} S^{*}}{N^{*}} \\
\Rightarrow h_{5}(\varpi)=y_{5} \varpi^{5}+y_{4} \varpi^{4}+y_{3} \varpi^{3}+y_{2} \varpi^{2}+y_{1} \varpi+y_{0}=0
\end{gathered}
$$

Where, $y_{5}=-1, y_{4}=\left(f+q-c_{2}-c_{3}-c_{4}\right)$

$$
\begin{gathered}
y_{3}=\left(-g l-f q+\varepsilon \omega+c_{3}\left(f+q-c_{4}\right)+c_{2}\left(f+q-c_{3}-c_{4}\right)+f c_{4}+q c_{4}+r \xi_{1}+m \xi_{2}+n \xi_{3}\right) \\
y_{2}=\left(-f \varepsilon \omega-q \varepsilon \omega-g l c_{4}-f q c_{4}-f r \xi_{1}-g r \xi_{1}+n \varepsilon \xi_{1}-f m \xi_{2}-g m \xi_{2}+m c_{4} \xi_{2}\right. \\
+c_{3}\left(-g l-f q+(f+q) c_{4}+r \xi_{1}+m \xi_{2}\right)-f n \xi_{3}-g n \xi_{3}+r \omega \xi_{3}+n c_{4} \xi_{3} \\
\left.+c_{2}\left(-g l-f q+\varepsilon \omega+c_{3}\left(f+q-c_{4}\right)+(f+q) c_{4}+r \xi_{1}+n \xi_{3}\right)+r \xi_{2} \tau_{1}+n \xi_{2} \tau_{2}\right) \\
y_{1}=\left(g l \varepsilon \omega+f q \varepsilon \omega-f n \varepsilon \xi_{1}-g n \varepsilon \xi_{1}-m \varepsilon \omega \xi_{2}-f m c_{4} \xi_{2}-g m c_{4} \xi_{2}-f r \omega \xi_{3}-g r \omega \xi_{3}-f n c_{4} \xi_{3}-g n c_{4} \xi_{3}-c_{2}(f \varepsilon \omega\right. \\
+q \varepsilon \omega+f r \xi_{1}+g r \xi_{1}-n \varepsilon \xi_{1}+c_{3}\left(g l+f q-(f+q) c_{4}-r \xi_{1}\right)+f n \xi_{3}+g n \xi_{3}-r \omega \xi_{3}+c_{4}(g l+f q \\
\left.\left.-n \xi_{3}\right)\right)-f r \xi_{2} \tau_{1}-g r \xi_{2} \tau_{1}+n \varepsilon \xi_{2} \tau_{1}-c_{3}\left((f+g) r \xi_{1}+c_{4}\left(g l+f q-m \xi_{2}\right)+\xi_{2}\left((f+g) m-r \tau_{1}\right)\right) \\
\left.-f n \xi_{2} \tau_{2}-g n \xi_{2} \tau_{2}+r \omega \xi_{2} \tau_{2}+n c_{4} \xi_{2} \tau_{2}\right) \\
\quad+(f+g) \xi_{2}\left(m \varepsilon \omega-n \varepsilon \tau_{1}-c_{3}\left(m c_{4}+r \tau_{1}\right)-r \omega \tau_{2}-n c_{4} \tau_{2}\right)
\end{gathered}
$$

The endemic steady state is locally asymptotically stable, if the necessary and sufficient conditions that all the roots of the polynomial in (33) have negatives real parts are all the polynomial coefficients must have the same sign (all negative in this case), nonzero. In addition, the first column containing the term $y_{5}$ of the Routh's array should be positive by Routh-Hurwitz stability criterion.

\subsection{Global Stability of the Endemic Equilibrium Point}

Theorem 10. Endemic equilibrium point $\left(E_{h}\right)$ is globally asymptotically stable, if $R_{h}>1$

Proof: By applying Lyapunov theorem,

Let $g(\zeta)=\zeta-1-\ln \left(\frac{1}{\zeta}\right), \zeta \in R^{+}$. Define a Lyapunov function 


$$
\begin{gathered}
V\left(S, H_{1}, H_{2}, A, T_{H}\right)=S^{*} g\left(\frac{S}{S^{*}}\right)+H_{1}^{*} g\left(\frac{H_{1}}{H_{1}^{*}}\right)+H_{2}^{*} g\left(\frac{H_{2}}{H_{2}^{*}}\right)+A^{*} g\left(\frac{A}{A^{*}}\right)+T_{H}^{*} g\left(\frac{T_{H}}{T_{H}^{*}}\right) \\
=\left[S-S^{*}-S^{*} \ln \left(\frac{S^{*}}{S}\right)\right]+\left[H_{1}-H_{1}^{*}-H_{1}^{*} \ln \left(\frac{H_{1}^{*}}{H_{1}}\right)\right]+\left[H_{2}-H_{2}^{*}-H_{2}^{*} \ln \left(\frac{H_{2}^{*}}{H_{2}}\right)\right] \\
+\left[A-A^{*}-A^{*} \ln \left(\frac{A^{*}}{A}\right)\right]+\left[T_{H}-T_{H}^{*}-T_{H}^{*} \ln \left(\frac{T_{H}^{*}}{T_{H}}\right)\right] \\
\frac{\partial V}{\partial S}=1-\frac{S^{*}}{S}, \frac{\partial V}{\partial H_{1}}=1-\frac{H_{1}{ }^{*}}{H_{1}}, \frac{\partial V}{\partial H_{2}}=1-\frac{H_{2}{ }^{*}}{H_{2}}, \frac{\partial V}{\partial A}=1-\frac{A^{*}}{A} \text { and } \frac{\partial V}{\partial T_{H}}=1-\frac{T_{H}{ }^{*}}{T_{H}},
\end{gathered}
$$

are all continuous partial derivatives for $\left(S, H_{1}, H_{2}, A, T_{H}\right) \neq$ value of $f$. Thus, $V\left(S^{*}, H_{1}^{*}, H_{2}^{*}, A^{*}, T_{H}^{*}\right)=0$ is minimum 0 .

functional value. Hence $V\left(S, H_{1}, H_{2}, A, T_{H}\right) \geq 0$.

Let $f(x)=x-u-u \ln \left(\frac{u}{x}\right)$, then for $x=u, f(x)=0$

The derivative of $V$ along the solution of the model is and for $x>u, f(x)>0$, and also $f(u)=0$ is the minimum

$$
\begin{gathered}
\frac{d V}{d t}=\frac{\partial V}{\partial S} \frac{d S}{d t}+\frac{\partial V}{\partial H_{1}} \frac{d H_{1}{ }^{*}}{d t}+\frac{\partial V}{\partial H_{2}} \frac{d H_{2}{ }^{*}}{d t}+\frac{\partial V}{\partial A} \frac{d A^{*}}{d t}+\frac{\partial V}{\partial T_{H}} \frac{d T_{H}{ }^{*}}{d t} \\
\frac{d V}{d t}=\Lambda-\frac{\beta\left(H_{1}+\psi_{2} H_{2}+\psi_{A} A+\psi_{H} T_{H}\right)}{N} S-\mu S-\frac{S^{*}}{S} \Lambda+\frac{S^{*}}{S} \frac{\beta\left(H_{1}+\psi_{2} H_{2}+\psi_{A} A+\psi_{H} T_{H}\right)}{N} S+\frac{S^{*}}{S} \mu S \\
+\frac{\beta\left(H_{1}+\psi_{2} H_{2}+\psi_{A} A+\psi_{H} T_{H}\right)}{N} S-\left(\xi_{1}+\xi_{2}+\xi_{3}+\mu\right) H_{1}-\frac{H_{1}^{*}}{H_{1}} \frac{\beta\left(H_{1}+\psi_{2} H_{2}+\psi_{A} A+\psi_{H} T_{H}\right)}{N} S \\
+\frac{H_{1}^{*}}{H_{1}}\left(\xi_{1}+\xi_{2}+\xi_{3}+\mu\right) H_{1}+\xi_{2} H_{1}-\left(\tau_{1}+\tau_{2}+\mu\right) H_{2}-\frac{H_{2}^{*}}{H_{2}} \xi_{2} H_{1}+\frac{H_{2}^{*}}{H_{2}}\left(\tau_{1}+\tau_{2}+\mu\right) H_{2} \\
+\xi_{3} H_{1}++\tau_{2} H_{2}+\varepsilon T_{H}-(\omega+\gamma+\mu) A-\frac{A^{*}}{A} \xi_{3} H_{1}-\frac{A^{*}}{A} \tau_{2} H_{2}-\frac{A^{*}}{A} \varepsilon T_{H}+\frac{A^{*}}{A}(\omega+\gamma+\mu) A \\
+\xi_{1} H_{1}+\tau_{1} H_{2}+\omega A-(\varepsilon+\mu) T_{H}-\frac{T_{H}^{*}}{T_{H}} \xi_{1} H_{1}-\frac{T_{H}^{*}}{T_{H}} \tau_{1} H_{2}-\frac{T_{H}^{*}}{T_{H}} \omega A+\frac{T_{H}^{*}}{T_{H}}(\varepsilon+\mu) T_{H}
\end{gathered}
$$

\begin{tabular}{|c|c|c|c|}
\hline Parameter & Description & Value & Reference \\
\hline$N$ & Total human population & 1000 & {$[4]$} \\
\hline$\Lambda$ & Recruitment rate & $\frac{1000}{65 \times 365}$ & [4] \\
\hline$\mu$ & Death rate of unrelated HIV/AIDS & $\frac{1}{65 \times 365}$ & [4] \\
\hline$\xi_{1}$ & Rate of ART treatment on acute stage & $0.01\left(\right.$ day $\left.^{-1}\right)$ & [4] \\
\hline$\xi_{2}$ & Rate of transition to pre-AIDS (Chronic) stage & $\frac{1}{5 \times 365}$ & [4] \\
\hline$\xi_{3}$ & $\begin{array}{l}\text { Rate of transition to full blown AIDS stage from acute stage } \\
\text { Rate of ART treatment on pre-AIDS stag }\end{array}$ & $\begin{array}{l}0.00055\left(d^{2} a y^{-1}\right) \\
0.01\left(d^{-1}\right)\end{array}$ & {$[12]$} \\
\hline $\begin{array}{l}\tau_{1} \\
\tau_{2}\end{array}$ & Rate of movement to full blown AIDS from pre-AIDS & $\frac{1}{3 \times 365}$ & [4] \\
\hline
\end{tabular}

We let $W=\Lambda+\frac{S^{*}}{S} \frac{\beta\left(H_{1}+\psi_{2} H_{2}+\psi_{A} A+\psi_{H} T_{H}\right)}{N} S+\frac{S^{*}}{S} \mu S+\frac{\beta\left(H_{1}+\psi_{2} H_{2}+\psi_{A} A+\psi_{H} T_{H}\right)}{N} S+\frac{H_{1}^{*}}{H_{1}}\left(\xi_{1}+\xi_{2}+\xi_{3}+\mu\right) H_{1}+\frac{H_{2}^{*}}{H_{2}}\left(\tau_{1}+\tau_{2}+\right.$ $\mu) H_{2}+\frac{A^{*}}{A}(\omega+\gamma+\mu) A+\xi_{1} H_{1}+\tau_{1} H_{2}+\omega A+\frac{T_{H}^{*}}{T_{H}}(\varepsilon+\mu) T_{H}$ and

$$
\begin{gathered}
Z=\frac{\beta\left(\mathrm{H}_{1}+\psi_{2} \mathrm{H}_{2}+\Psi_{\mathrm{A}} \mathrm{A}+\Psi_{\mathrm{H}} \mathrm{T}_{\mathrm{H}}\right)}{\mathrm{N}} \mathrm{S}+\mu \mathrm{S}+\frac{\mathrm{S}^{*}}{\mathrm{~S}} \Lambda+\left(\xi_{1}+\mu\right) \mathrm{H}_{1}+\frac{\mathrm{H}_{1}^{*}}{\mathrm{H}_{1}} \frac{\beta\left(\mathrm{H}_{1}+\psi_{2} \mathrm{H}_{2}+\Psi_{\mathrm{A}} \mathrm{A}+\psi_{\mathrm{H}} \mathrm{T}_{\mathrm{H}}\right)}{\mathrm{N}} \mathrm{S} \\
+\left(\tau_{1}+\mu\right) \mathrm{H}_{2}+\frac{\mathrm{H}_{2}^{*}}{\mathrm{H}_{2}} \xi_{2} \mathrm{H}_{1}+(\omega+\gamma+\mu) \mathrm{A}+\frac{\mathrm{A}^{*}}{\mathrm{~A}} \xi_{3} \mathrm{H}_{1}+\frac{\mathrm{A}^{*}}{\mathrm{~A}} \tau_{2} \mathrm{H}_{2}+\frac{\mathrm{A}^{*}}{\mathrm{~A}} \varepsilon \mathrm{T}_{\mathrm{H}}+(\varepsilon+\mu) \mathrm{T}_{\mathrm{H}}+\frac{\mathrm{T}_{\mathrm{H}}^{*}}{\mathrm{~T}_{\mathrm{H}}} \xi_{1} \mathrm{H}_{1}+\frac{\mathrm{T}_{\mathrm{H}}^{*}}{\mathrm{~T}_{\mathrm{H}}} \tau_{1} \mathrm{H}_{2}+\frac{\mathrm{T}_{\mathrm{H}}^{*}}{\mathrm{~T}_{\mathrm{H}}} \omega \mathrm{A}
\end{gathered}
$$

Then, $\frac{d V}{d t}=W-Z$ and $\frac{d V}{d t} \leq 0$ if $W<Z$.

$\frac{d V}{d t}=0$ if and only if $\left(S, H_{1}, H_{2}, A, T_{H}\right)=\left(S^{*}, H_{1}^{*}, H_{2}^{*}, A^{*}, T_{H}^{*}\right)$.

The largest compact invariant subsets in $\left\{\left(S, H_{1}, H_{2}, A, T_{H}\right)\right\}$ is the singleton $\operatorname{set}\left\{\mathrm{E}_{\mathrm{h}}\right\}$. Therefore, by Lasalle's invariant principle (Lasalle 1976), $\mathrm{E}_{\mathrm{h}}$ is global attractor whenever $R_{h}>1$.

Table 1. Definition and parameter values for the model. 


\begin{tabular}{llll}
\hline Parameter & Description & Value \\
\hline$\varepsilon$ & Rate of transition for the failure of treatment & $\frac{0.5}{365}$ & Reference \\
$\omega$ & Rate of ART treatment on AIDS stage & $0.01\left(\right.$ day $\left.^{-1}\right)$ \\
$\gamma$ & Death rate due to HIV/AIDS & $0.00008($ day & \\
$\beta$ & Probability of infection of HIV by acute stage & 0.0025 & {$[4]$} \\
$\psi_{2}$ & Modification parameter for the infectiousness in class $H_{2}$ & 0.75 & {$[4]$} \\
$\psi_{A}$ & Modification parameter for the infectiousness in class $A$ & 0.5 \\
$\psi_{H}$ & Modification parameter for the infectiousness in class $T_{H}$ & 0.001 \\
\hline
\end{tabular}

\subsection{Sensitivity Analysis of the Effective Reproduction Number}

To conduct the sensitivity analysis, we adopt the normalized forward sensitivity index. The partial derivative is the rate of change of prediction with respect to each parameter using the approach in (Chitns et al 2008), (Edward and Nyerere 2015), (Numfor 210). The degree of sensitivity index of the reproduction number with respect to a parameter say $h$, measures the relative change in variable when the parameter $g$ changes as $\varphi_{g}^{R_{h}}=\frac{\partial R_{h}}{\partial g}\left(\frac{g}{R_{h}}\right)$. The value $\varphi_{g}^{R_{h}}=1$, shows an increase of $g$ results in an increase of $R_{h}$ and a decrease of $g$ results in decrease of $R_{h}$ and $\varphi_{g}^{R_{h}}=-1$, shows an increase of $g$ results in a decrease of $R_{h}$ and a decrease of $g$ results in an increase of $R_{h}$. In our case, we calculate the sensitivity indices of the parameters using the values of the parameters from different literatures in table 1 .

First let us analyse $R_{h}$, to understand whether implementing treatment of ART in the acute stage (with rate $\xi_{1}$ ), in pre AIDS stage (with rate $\tau_{1}$ ), in the AIDS stage (with rate $\omega$ ) on a single stage or simultaneously in all stages leads to control the spread of HIV in the community effectively. It is true that from the effective reproduction number $R_{h}$

$$
\begin{gathered}
\lim _{\xi_{1} \rightarrow \infty} R_{h}=\frac{\beta \psi_{A}\left(\varepsilon\left(\tau_{1}+\tau_{2}+\mu\right)\right)+\beta \psi_{H}\left(\left(\tau_{1}+\tau_{2}+\mu\right)(\omega+\gamma+\mu)\right)}{\left(\tau_{1}+\tau_{2}+\mu\right)[-\varepsilon \omega+(\omega+\gamma+\mu)(\varepsilon+\mu)]}>0 \\
\lim _{\tau_{1} \rightarrow \infty} R_{h}=\frac{\left.\beta \psi_{A}\left((\varepsilon+\mu) \xi_{3}+\varepsilon \xi_{1}+\varepsilon \xi_{2}\right)\right)+\beta \psi_{H}\left(\omega \xi_{3}+(\omega+\gamma+\mu) \xi_{1}+(\omega+\gamma+\mu) \xi_{2}\right)}{\left(\xi_{1}+\xi_{2}+\xi_{3}+\mu\right)[-\varepsilon \omega+(\omega+\gamma+\mu)(\varepsilon+\mu)]}>0 \\
\lim _{\omega \rightarrow \infty} R_{h}=\frac{\beta \psi_{2}\left(-\varepsilon \xi_{2}+(\varepsilon+\mu) \xi_{2}\right)+\beta \psi_{H}\left(\left(\tau_{1}+\tau_{2}+\mu\right) \xi_{3}+\left(\tau_{1}+\tau_{2}+\mu\right) \xi_{1}+\xi_{2} \tau_{1}+\xi_{2} \tau_{2}\right)}{\left(\xi_{1}+\xi_{2}+\xi_{3}+\mu\right)\left(\tau_{1}+\tau_{2}+\mu\right) \mu}>0
\end{gathered}
$$

Thus, effective HIV treatment that targets treating individuals in acute stage with high rate $\left(\xi_{1} \rightarrow \infty\right)$ or treating individuals in pre-AIDS stage with rate $\left(\tau_{1} \rightarrow \infty\right)$ or treating individual in AIDS stage with rate $(\omega \rightarrow \infty)$ could lead to effective control of HIV transmission, if all the above limits are less than unity. That is respectively if,

$$
\begin{gathered}
\beta \psi_{A}\left(\varepsilon\left(\tau_{1}+\tau_{2}+\mu\right)\right)+\beta \psi_{H}\left(\left(\tau_{1}+\tau_{2}+\mu\right)(\omega+\gamma+\mu)\right) \\
<\left(\tau_{1}+\tau_{2}+\mu\right)[-\varepsilon \omega+(\omega+\gamma+\mu)(\varepsilon+\mu)], \\
\left.\beta \psi_{A}\left((\varepsilon+\mu) \xi_{3}+\varepsilon \xi_{1}+\varepsilon \xi_{2}\right)\right)+\beta \psi_{H}\left(\omega \xi_{3}+(\omega+\gamma+\mu) \xi_{1}+(\omega+\gamma+\mu) \xi_{2}\right. \\
<\left(\xi_{1}+\xi_{2}+\xi_{3}+\mu\right)[-\varepsilon \omega+(\omega+\gamma+\mu)(\varepsilon+\mu)]
\end{gathered}
$$

and $\beta \psi_{2}\left(-\varepsilon \xi_{2}+(\varepsilon+\mu) \xi_{2}\right)+\beta \psi_{H}\left(\left(\tau_{1}+\tau_{2}+\mu\right) \xi_{3}+\left(\tau_{1}+\tau_{2}+\mu\right) \xi_{1}+\xi_{2} \tau_{1}+\xi_{2} \tau_{2}<\left(\xi_{1}+\xi_{2}+\xi_{3}+\mu\right)\left(\tau_{1}+\tau_{2}+\mu\right) \mu\right.$

Secondly, let us try to answer the question that, at which stage of HIV/AIDS, ART treatment should be implemented to control the spread of HIV in the community effectively? To answer this question, first we target treatment of ART for individuals at every stage of HIV/AIDS. $\left(\xi_{1} \neq 0, \tau_{1} \neq 0, \omega \neq 0\right)$.

$$
\begin{gathered}
c_{1}=\xi_{1}+\xi_{2}+\xi_{3}+\mu=0.01114, c_{2}=\tau_{1}+\tau_{2}+\mu=0.01094 \\
c_{3}=\omega+\gamma+\mu=0.01012, c_{4}=\varepsilon+\mu=0.00141 \\
n_{1}=c_{2} c_{3} c_{4}-\varepsilon \omega c_{2}=6.227048 \times 10^{-9}, n_{2}=\xi_{2}\left(c_{3} c_{4}-\varepsilon \omega\right)=3.1295 \times 10^{-10} \\
n_{4}=c_{2} c_{4} \xi_{3}+\varepsilon c_{2} \xi_{1}+\varepsilon \xi_{2} \tau_{1}+c_{4} \xi_{2} \tau_{2}=1.6658 \times 10^{-7} \\
n_{8}=\omega c_{2} \xi_{3}+c_{2} c_{3} \xi_{1}+c_{3} \xi_{2} \tau_{1}+\omega \xi_{2} \tau_{2}=0.0000012279 \\
D=-\varepsilon \omega c_{1} c_{2}+c_{1} c_{2} c_{3} c_{4}=6.9369 \times 10^{-11} \\
n_{1}+\psi_{2} n_{2}+\psi_{A} n_{4}+\psi_{H} n_{8}=n_{1}+0.75 n_{2}+0.5 n_{4}+0.001 n_{8}=9.09796 \times 10^{-8} \\
\mathrm{R}_{\mathrm{h}}=\beta\left(\frac{n_{1}+\psi_{2} n_{2}+\psi_{A} n_{4}+\psi_{H} n_{8}}{D}\right)=\frac{2.27449 \times 10^{-10}}{6.9369 \times 10^{-11}}=\frac{22.7449}{6.9369}=3.2788
\end{gathered}
$$


Let us target the treatment of ART is only on acute stage alone $\left(\tau_{1}=\omega=0\right)$

$$
\begin{gathered}
c_{1}=\xi_{1}+\xi_{2}+\xi_{3}+\mu=0.01114, c_{2}^{*}=\tau_{2}+\mu=0.00094 \\
c_{3}^{*}=\gamma+\mu==0.00012, c_{4}^{*}=\varepsilon+\mu=0.00141
\end{gathered}
$$

Considering treatment target only on a single stage acute $\left(H_{1}\right)\left(\right.$ i.e $\left.\tau_{1}=\omega=0\right)$

$$
\begin{gathered}
n_{1}^{*}=1.59 \times 10^{-10}, n_{2}^{*}=9.306 \times 10^{-11}, n_{4}^{*}=1.4304 \times 10^{-8}, n_{8}^{*}=1.128 \times 10^{-9}, D^{*}=1.7717 \times 10^{-12} \\
n_{1}^{*}+\psi_{2} n_{2}^{*}+\psi_{A} n_{4}^{*}+\psi_{H} n_{8}^{*}= \\
n_{1}+0.75 n_{2}+0.5 n_{4}+0.001 n_{8}=7.382 \times 10^{-9} \\
R^{*}{ }_{\mathrm{h}}=\beta\left(\frac{n_{1}^{*}+\psi_{2} n_{2}^{*}+\psi_{A} n_{4}^{*}+\psi_{H} n_{8}^{*}}{D^{*}}\right)=10.4165
\end{gathered}
$$

From these results, we can observe that strategy that focuses on treating individuals at every stage reduces the basic reproduction number (Theorem 10) from $\mathrm{R}_{0 \mathrm{~h}}=13$ to $R_{h}=3$, whereas, the second strategy that focuses on treating individuals only in acute stage alone reduces the basic reproduction number from $\mathrm{R}_{0 \mathrm{~h}}=13$ to $R_{\mathrm{h}}^{*}=10$, Thus, HIV can not be eliminated by implementing the second strategy. Similarly, implementing treatment on any single stage of HIV/AIDS alone is not effective to control the spread of HIV. In general, the first strategy that targets on treating individuals at every stage reduces reproduction number faster than implementing at one stage alone and hence leads to elimination of HIV/AIDS in the community (verified by figure $2 \mathrm{~A}$ and $2 \mathrm{~B}$ ). Therefore, infected individuals should be treated at every stage of HIV/AIDS and hence all treatment parameters are very important to reduce the magnitude of reproduction number.

Table 2. Sensitivity indices of the effective reproduction number to model parameters.

\begin{tabular}{ll}
\hline Parameter & Sensitivity index \\
\hline $\mathrm{B}$ & 1 \\
$\mathrm{E}$ & 0.64545 \\
$\xi_{1}$ & -0.061845 \\
$\xi_{2}$ & -0.0008786 \\
$\xi_{3}$ & -0.0020858 \\
$\tau_{2}$ & -0.0011675 \\
$\Gamma$ & -0.18435 \\
\hline
\end{tabular}

From the values of sensitivity indices in table 2 , we can see that an increase in transmission rate of $\operatorname{HIV}(\beta)$ will increases the infective (acute stage) population. In addition, an increase in waning (failing) of ART treatment rate $(\varepsilon)$ will increase the AIDS population. Thus, HIV transmission rate and ART treatment waning rate have positive impact on the magnitude of effective reproduction number. An increase in transition rate from infective (acute) stage to treated, pre-AIDS and AIDS stages, decrease the infective population which in turn increase the treated population. As movement from infective increases, the infective population decreases and hence the pre-AIDS and AIDS population decrease with time until they reach the equilibrium point.

On the other hand, HIV/AIDS related mortality rate $(\gamma)$, ART treatment rate $\left(\xi_{1}\right)$ of HIV/AIDS have negative influence on the magnitude of reproduction number. However, we cannot control HIV prevalence by disease mortality, since our objective is saving life. An increase in ART treatment control strategy has positive impact in controlling HIV transmission and hence we can reduce the transition to pre-AIDS and AIDS classes by high rate of treatment. The transition rate $\left(\tau_{2}\right)$ from pre-AIDS to AIDS class has again negative impact on the magnitude of reproduction number, but we can reduce this transition by increasing the ART treatment rate $\left(\tau_{1}\right)$ on pre-AIDS class.

From the sensitivity index in table 2 , the transmission rate of HIV $(\beta)$, treatment rate $\left(\xi_{1}\right)$ and rate of failure of ART treatment $(\varepsilon)$ are highest in magnitude, thus, effective reproduction number is more sensitive to these parameters.

\section{Numerical Simulation}

We carried out numerical simulations to verify and support the impact of basic model parameters on the reproduction number, using set of model parameters whose values are taken from literature. The model parameter values and respective sources are present in table 1.

The basic reproduction number $R_{o h}$, of the dynamics without public health intervention strategy based on the standard data from literature is

$$
\mathrm{R}_{0 \mathrm{~h}}=\frac{\beta}{\xi_{2}+\xi_{3}+\mu}\left(1+\frac{\psi_{2} \xi_{2}}{\tau_{2}+\mu}+\frac{\psi_{A} \xi_{3}}{\gamma+\mu}+\frac{\psi_{A} \xi_{2} \tau_{2}}{\left(\tau_{2}+\mu\right)(\gamma+\mu)}\right)=13
$$

Whereas the effective reproduction number $R_{h}$ with control strategy is given as

$$
\mathrm{R}_{\mathrm{h}}=\beta\left(\frac{n_{1}+\psi_{2} n_{2}+\psi_{A} n_{4}+\psi_{H} n_{8}}{D}\right)=3.2788
$$

From the above results, the basic reproduction number tells us a single HIV infected individual can generate about 13 new secondary infections in the community during his/her entire infection period in a completely susceptible population in the absence of HIV treatment and continuous education campaign on ART. The effective reproduction number tells us about 3 new infections were generated by a single infected individual in the community where ART treatment intervention, targeted at every stage of HIV/AIDS and continuous education campaign using different medias about the correct procedure of ART treatment in treated class are implemented. Thus, we can observe that $R_{h}<<R_{0 h}$ and our public health intervention strategies are effective to control the spread of 
HIV/AIDS infection.

$$
\mathrm{R}_{h}(\beta)=(1,311.5) \beta
$$

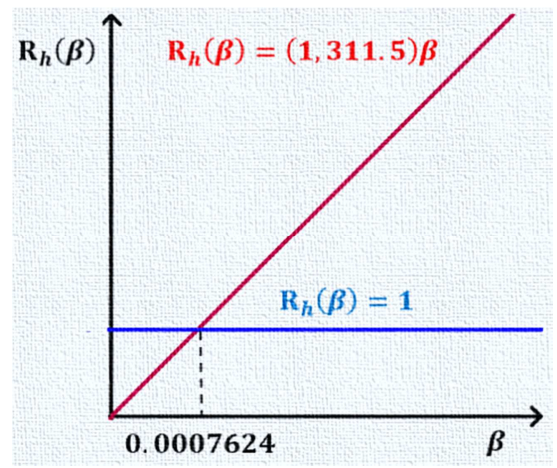

Figure 2. Effective reproduction number $R_{h}$ versus HIV transmission rate $\beta$.

From figure 1, HIV transmission rate $(\beta)$ reduces the magnitude of reproduction number to the values less than unity with rate lesssthan 0.0007624 and reproduction number increases as transmission rate increases.

Consider the case treatment targets only in acute stage (i.e $\tau_{1}=\omega=0$ ).

Thus,

$$
\mathrm{R}_{\mathrm{h}}\left(\xi_{1}\right)=\frac{0.0123115+10.1229 \xi_{1}}{\xi_{1}+0.00114}
$$

Consider the case treatment targets on every stage of HIV/AIDS (i.e $\xi_{1} \neq 0, \tau_{1} \neq 0 . \omega \neq 0$ ).

Thus,

$$
\mathrm{R}_{\mathrm{h}}\left(\xi_{1}\right)=\frac{3.05305 \xi_{1}+0.003498}{\xi_{1}+0.00114}
$$

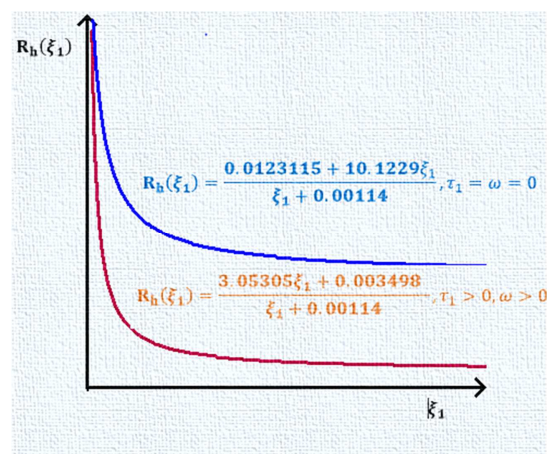

Figure 3. Effective reproduction number $R_{h}$ versus ART treatment rate in acute stage, $\xi_{1}$ as variable, when $\tau_{1}=\omega=0$ and when $\tau_{1}$ and $\omega$ are non-zero positive constants.

From figure 2, when treatment intervention is implemented only in acute stage, the effective reproduction number reduce slowly. As the treatment increases the reproduction number decreases. But when treatment intervention is implemented in all stage of HIV/AIDS, it reduces the reproduction number faster than intervention only in acute stag. In both cases, the graph supports the analytical result that treatment only in one stage and simultaneous treatment in all stages of HIV/AIDS has negative impact on the magnitude of effective reproduction number $R_{h}$, however simultaneous treatment in all stages is very much important in eliminating the spread of the disease in the community.

$$
R_{h}(\varepsilon)=\frac{15.51 \varepsilon+0.0014}{1.46 \varepsilon+0.49}
$$

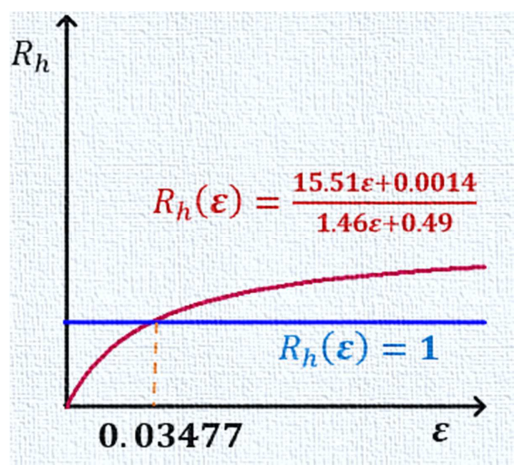

Figure 4. Effective reproduction number $R_{h}$ versus ART treatment waning rate $\varepsilon$.

From figure 3, If the rate of failure of treatment due to improper use of procedure of ART treatment increases, the magnitude of the reproduction number also increases. In particular if the failure of the treatment rate is greater than 0.03477 , reproduction number is above unity (disease transmission increase in the community). The reproduction number is below unity when the treatment waning (failing) rate is between 0 and 0.03477 (disease dies out in the community).

$$
\mathrm{R}_{\mathrm{h}}(\gamma)=\frac{0.0081+3.89 \gamma}{0.0055+17.18 \gamma}
$$

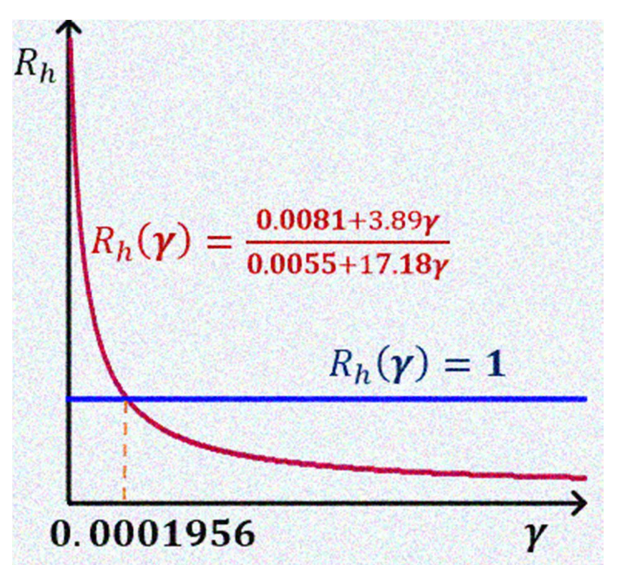

Figure 5. Effective reproduction number $R_{h}$ versus AIDS induced death rate $\gamma$.

From figure 4, when disease induced death rate increase, the effective reproduction number decrease and less than unity for $\gamma>0.0001956$. This verifies that AIDS induced death rate has negative impact on the magnitude of effective reproduction number, but we cannot reduce the effective reproduction number by killing people as our objective is saving life. However, it is true that AIDS induced death rate, can be controlled by ART treatment together with continuous education campaign on the proper procedure of ART treatment. 


\section{Discussion}

Human immunodeficiency virus (HIV) infection is a disease of immune system caused by HIV virus and transmitted primarily via unprotected sexual intercourse, contaminated blood transfusions (Horizontal transmission) and from mother to child during pregnancy, delivery or breastfeeding (Vertical transmission).

In this paper, we proposed a deterministic mathematical model, incorporating public health interventions ART treatment, which is, targeted at every stage of HIV/AIDS and continuous education campaign on the proper procedure of ART treatment in the transmission dynamics of HIV infection. The population is grouped in to Susceptible $(S)$, Acute stage $\left(H_{1}\right)$, Pre-AIDS stage $\left(H_{2}\right)$, AIDS stage $(A)$ and Treated individuals are in compartment $\left(T_{H}\right)$ by modifying $\mathrm{SH}_{1} \mathrm{H}_{2} \mathrm{~A}$ model, where treatment is targeted only on pre-AIDS stage and individuals in pre-AIDS class will return back to acute stage by the help of treatment. In most researches like in $[4,11]$ awareness on the proper procedure of ART treatment is not considered as a major problem for the failure of treatment. In our research, we tried to understand the effect of control measures treatment and education campaign on ART with only horizontal transmission way of HIV, to gain useful strategies to the effective prevention and intervention against HIV prevalence. The basic reproduction number $\left(R_{0 h}\right)$ and the effective reproduction number $\left(R_{h}\right)$ are determined by applying the next generation approach in Diekmann et al. 1990 [23]. The disease free $\left(E_{0 h}\right)$ and endemic $\left(E_{h}\right)$ equilibria are indicated to be locally and globally asymptotically stable for $R_{h}<1$ and $R_{h}>1$ respectively in the mathematical results. This shows that HIV/AIDS dies out in the community, if the treatment rate and treatment waning rate bring $\left(R_{h}\right)$ less than unity and the disease persist in the community, if $R_{h}>1$. We have shown that $R_{h}<R_{0 h}$, and $R_{h}=R_{0 h}$ when the treatment rate and treatment waning rate equal to zero $\left(\xi_{1}=\tau_{1}=\omega=\varepsilon=0\right)$. This mean that treatment rate and education campaign to decrease ART waning rate will reduce the basic reproduction number and thus they are important to control HIV transmission. On the other hand, in the absence of these two interventions the spread of the disease will be high.

By evaluating the sensitivity indices of the effective reproduction number with respect to model parameters, the influential parameters for the spread of the disease are identified and thus HIV transmission rate, ART treatment rate and ART waning rate are influential parameters. The most influential one is HIV transmission rate. From the values of sensitivity indices, an increase HIV transmission rate and increase in failure of treatment rate may increase the magnitude of reproduction number. On the other hand, HIV/AIDS related mortality, ART treatment, movement to acute stage, movement to pre-AIDS and full-blown AIDS stages have negative influence on the magnitude of reproduction number. The movement from acute stage to pre-AIDS and AIDS stages can be control by high rate of ART treatment on infective (Acute stage). The transmission rate of HIV can be reduced by education campaign on the danger of
HIV/AIDS primary. However, we cannot control HIV prevalence by disease and natural mortality, since our objective is preventing people from dying of the disease and save life. Therefore, sensitivity analysis helps to focus on high sensitive parameters of the reproduction number in order to combat the transmission with low cost, if there is economic constrained. Both ART treatment and continuous education campaign on ART treatment (to decrease the failure of ART) had positive impact in reducing the disease prevalence. Moreover, numerical simulations support the analytical results, that both in an increase in ART treatment and continuous education campaign on the proper procedure of ART treatment (to reduce the rate of failure of treatment) will reduce the magnitude of the basic reproduction number

\section{Conclusion and Recommendation}

We rigorously analysed a deterministic mathematical model of the transmission of HIV virus in a population with treatment and continuous education campaign on the proper procedure of ART treatment (to reduce the failure of treatment rate). We have seen that there exists a feasible region where the model is well posed and a unique disease free steady state exists in the region.

The disease free and endemic equilibria were obtained and their stabilities investigated. It was established that the disease free and endemic equilibria are locally and globally asymptotically stable for the effective reproduction number $R_{h}<1$ and $R_{h}>1$ respectively. This shows that HIV/AIDS dies out in the community, if the effective reproduction number is less than unity and persist otherwise. Moreover, based on the parameter values, we have $R_{h}<R_{0}$. This mean that both ART treatment and continuous education campaign on procedure of ART treatment will reduce the basic reproduction number. That is implementing treatment with high rate will reduce the movement from infective (acute stage) to pre-AIDS and full blown AIDS stages and an increase continuous education campaign on the proper procedure of ART treatment through different medias will reduce the failure of ART treatment, with this we can control the transmission of HIV.

In addition, from the numerical simulation, by increasing treatment rate, controlling HIV transmission rate and failure of treatment rates, the spread of the disease can be reduced significantly and finally the disease die out in the community.

There are different ways of preventing new HIV infections such as Education campaign through different media about the danger of AIDS, providing condoms at a very low cost, creating awareness on correct use of condoms and designing Strategy of community-based management of sexually transmitted infections (STIs).

Once the disease emerge in the community, based on the findings of our research, proper implementation of preventive mechanisms, ART treatment and continuous education campaign on the proper procedure of ART treatment must be the major concern to control the spread of the disease. Therefore, we recommend for the stakeholders to design policies, planning, budgeting finance and resource allocations primarily focusing on prevention and then all possible public 
health interventions to combat against HIV transmission in the community.

\section{Acknowledgements}

First, I would like to thank my God for his great charity. Next, I want to express my appreciation to my advisor Purnachandra Rao Koya (Prof.) for his support on this work, department of mathematic in Debre Berhan University for its financial support and my friends who encouraged me in one way or another on this work.

\section{References}

[1] Alexander Kramer et al. Modern Infectious Disease Epidemiology. Springer Science+Business Media, LLC 2010.

[2] FMoH, November 2018. Guideline for HIV CARE/ART clinical mentoring in Ethiopia. (http://www.afro.who).

[3] Kathleen M. MacQueen Division of HIV/AIDS, National Center. (1994). The epidemiology of HIV Transmission: Trends, Structure and Dynamics. Annual Review of Anthropology, Vol. 23, pp. 509-526.

[4] Maimunah, Dipo Aldila. (2018). Mathematical model for HIV spreads control program with ART treatment. Journal of Physics: Conf. Series 974 (2018) 012035.

[5] S. Mushayabasa, C. P. BhunuIs. (2012). HIV infection associated with an increased risk for cholera? Insights from a mathematical model. BioSystems 109 (2012) 203-213AL.

[6] A Cristiana J. Silva and Delfim F. M. Torres. (2015). A TB-HIV/AIDS Coinfection model and optimal control treatment. Department of Mathematics, University of Aveiro, 3810- 193 Aveiro, Portugal.

[7] AAM Arafa, SZ Rida and Khalil. (2012). Fractional modeling dynamics of HIV and CD4+ T-cells during primary infection. Nonlinear Biomedical Physics 2012, 6: 1.

[8] Ofosuhene O. Apenteng; Noor Azina Ismail. (2019). Modelling the impact of migration on HIV persistency in Ghana. Statistics, Optimization and Information Computing, 7 (1), 55-65.

[9] Rebecca V. Culshaw, Ph.D. Mathematical Modeling of AIDS Progression: Limitations, Expectations, and Future Directions. Journal of American Physicians and Surgeons. Volume 11 Number 4 Winter 2006.
[10] Susan Cassels, PhD et al. (2008). Mathematical Models for HIV Transmission Dynamics: Tools for Social and Behavioral Science Research. J Acquir Immune Defic Syndr. 2008 March 1; 47 (Suppl 1): S34-S39.

[11] Abdallah S. Waziri, Estomih S. Massawe, Oluwole Daniel Makinde. (2012). Mathematical Modelling of HIV/AIDS Dynamics with Treatment and Vertical Transmission. Applied Mathematics 2012. 2 (3): 77-89.

[12] Epidemiology of HIV/AIDS. (En.wikipedia.org/wiki/Epidemiolog_of HIV/AIDS).

[13] Epidemiology of HIV/AIDS. (En.wikipedia.org/wiki/HIV/AIDS in Africa).

[14] Timothy L. Mah, Daniel T. Halperin. (2008). Concurrent Sexual Partnerships and the HIV Epidemics in Africa: Evidence to Move Forward. AIDS Behav (2010) 14: 11-16.

[15] UNAIDS. 2017, Joint United Nations Programme on HIV/AIDS.

[16] WANG Jun-jie Kathleen Heather Reilly, LUO Jing, WANG Ning. (2010). Dynamic mathematical models of HIV/AIDS transmission in China. Chin Med J (Engl). 123 (15): 2120-21272.

[17] FMOH. November, 2018. HIV prevention in Ethiopia National Road Map 2018-2020.

[18] FMOH. August, 2018, National Consolidated guidelines for comprehensive HIV prevention, care and treatment.

[19] Fatmawati and Hengki Tasman. (2016). An Optimal Treatment Control of TB-HIV Coinfection. International Journal of Mathematics and Mathematical Sciences Volume 2016, Article ID 8261208,11 pages.

[20] K. O. Okosun, O. D. Makinde, I. Takaidza. (2012). Impact of optimal control on the treatment of HIV/AIDS and screening of unaware infectives. Applied Mathematical Modelling 37 (2013) $3802-3820$.

[21] Oluwaseun Sharomi, Chandra N. Podder, Abba B. Gumel. (2008). Mathematical analysis of the transmission dynamics of HIV/TB coinfection in the presence of treatment. Mathematical biosciences and engineering, Volume 5, pp. 145-174 D (9).

[22] Zohragul Osman, Xamxinur Abdurahman. (2015). Stability Analysis of a Delayed HIV/AIDS Epidemic Model with Treatment and Vertical Transmission.

[23] Carlos Castillo-Chavez Zhilan Feng and Wenzhang Huang. (2001). On the computation of Ro and its role on global stability. N-1553 (607) 255-8103. 